\title{
Evaluation of Nitric Oxide in Lacunar Stroke and Young Healthy during Cerebrovascular Reactivity by Support Vector Machine
}

\author{
Yutthana Phimthong- \\ Ngam \\ Medical Engineering Graduate \\ Program, Faculty of \\ Engineering, Thammasat \\ University (Rangsit Campus), \\ Pathumthani, Thailand
}

\author{
Kannakorn Intharakham \\ Medical Engineering Graduate \\ Program, Faculty of \\ Engineering, Thammasat \\ University (Rangsit Campus), \\ Pathumthani, Thailand
}

\author{
Kesorn Suwanprasert \\ Department of Preclinical \\ Sciences, Faculty of Medicine, \\ Thammasat University \\ (Rangsit Campus), \\ Pathumthani, Thailand
}

\begin{abstract}
Lacunar stroke (LS) is known as lacunar infarction, which presents minor deficit in neurological finding but it actually causes stroke recurrent and death. High risk of stroke such as chronic hypertension is closely associated with LS. In this study, vasodilatory stimulus through cerebrovascular technique (30 sec breath- holding) was used to activate the vasomotor tone of cerebral arteries. Then, plasma nitric oxide as neurovascular mediator maintaining cerebral blood flow was assessed. The objective of this study is to determine the effective procedure for nitric oxide (NO) classification and to distinguish NO in young healthy and in LS. NO concentration was real time monitored by using electrochemistry method. Collected NO data are characterized and used as training data for classification model study. Effective model will be used for prediction of high risk, recurrent and follow up stroke as well as recovery from any intervention treatment. The classifier performance by each single kernel function presents that only radial basis function (RBF) has highest performance (94\% classified accuracy) compared with those in linear, polynomial, and sigmoid functions in experiment phase. Combined with highest performance, the hybrid model was developed and given $96 \%$ accuracy. This novel model is the best classifier for NO as neurovascular biomarker in LS. The findings suggest that low values of right parameters $\psi$ and $\lambda$ are able to improve performance of NO classification in LS. The novel hybrid model is the best giving the greatest classification accuracy for NO that plays a novel role as neuromodulator and neurovascular biomarker.
\end{abstract}

\section{General Terms}

Pattern Recognition, Classification, Feature Extraction

\section{Keywords}

Lacunar Stroke, Nitric Oxide, Cerebrovascular Reactivity, Support Vector Machine

\section{INTRODUCTION}

Lacunar stroke (LS) is defined as the sudden onset in one of the classic lacunar syndromes accompanied by an ischemic lesion on CT or MRI scan. Lacunar infarct is very small and deep infarcts $(2-15 \mathrm{~mm}$ in diameter) of small penetrating cerebral arterioles caused by lipohyalinosis and cerebral vascular inflammation from oxidative stress [1]. Several lines of evidence have reported that LS is closely related with recurrent stroke and death [2]. Brief 30 second - breath holding maneuver, a method of cerebrovascular reactivity (CVR) assessment, induces an increasing cerebral $\mathrm{pCO}_{2}$ (hypercapnia) promoting higher cerebral blood flow from vasodilation and reflects the compensatory dilatory capacity of cerebral arterioles[3]. An adequate cerebral blood flow is essential in cerebral reserve function through an autoregulation process. Previous study, NO plays a vital role in vasomotor tone of cerebral artery. In the healthy young age model, an increase in cerebral blood flow resulting from CVR is closely correlated with NO [4]. In cerebral artery, NO is produced by eNOS resulting phosphorylation of multiple proteins that causes smooth muscle relaxation [5]. Moreover, $\mathrm{NO}$ is also produced by nNOS activation of L-arginine in brain promoting an excitatory glutamate release at synaptic junction. Thus, NO is an important neurovascular modulator for brain activity and function [6]. Since a small change value of NO in lacunar and high risk of stroke is related to oxidative stress, classification of NO is very useful for neurovascular assessment and represents how the degree of cerebral reserve function. In this study, wide ranges of NO concentrations from young healthy to lacunar stroke for training and testing data are classified. The finding will be used and applied in the stroke risk forecast. Machine learning method is proposed to be a tool for NO classification.

Support Vector Machines (SVMs) are high-performance supervised machine learning techniques used in regression, classification, and other learning tasks [7]. In classification, SVMs are a powerful binary classifiers for solving binary problems in nonlinear classification. In physiological response, all data are mapped to a high-dimensional future space using a mapping function that separates the data into two classes within the maximum margin. The mapping function is called kernel function, which it is a key part of SVMs. Different kinds of kernel functions are wildly used and applied in order to obtain the meaningful results. SVMs modeling was firstly designated as the classification of linearly separable classes of data. For any specific set of twoclass objects, the SVMs find the unique hyperplane having the maximum margin (denoted with $\delta$ in Figure 1a). The main intuition is two classes training sets which they project their data points in a higher-dimensional space and attempt to specify a maximum-margin separating hyperplane between the data points into two classes. This hyperplane is optimal in the sense that it generalizes well to unseen data. The hyperplane $\mathrm{H} 1$ defines the border with class +1 objects, whereas the hyperplane $\mathrm{H} 2$ defines the border with class -1 objects. Two objects from class +1 define the hyperplane $\mathrm{H} 1$, and three objects from class -1 define the hyperplane $\mathrm{H} 2$. These objects, represent inside circles in Figure 1a, are named support vectors [8]. When a sample is linear separable, a 
hyperplane or a set of hyperplanes that separate the data is constructed. The samples from the class 1 that satisfy the equation are considered [9]:

$$
\begin{aligned}
& w^{T} x_{i}+b \geq 1 \text { when } y_{i}=1 \\
& w^{T} x_{i}+b \leq 1 \text { when } y_{i}=-1
\end{aligned}
$$

Subject to

$$
y_{i}\left(w^{T} x_{i}+b\right) \geq 1, \quad i=1,2, \ldots, N
$$

where $y_{i} \in\{-1,1\} \quad$ represent the class of negative and positive, $\mathrm{x}$ is input vector, $w$ is the weight vector, - $\mathrm{b}$ or $\mathrm{b}$ is the threshold and bias respectively, and $i$ is the number of trained data point. With the decision rule expresses as follows:

$$
f_{w, b}(x)=\operatorname{sgn}\left(w^{T} x+b\right)
$$

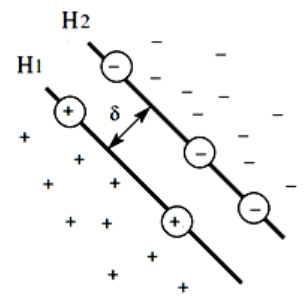

a)

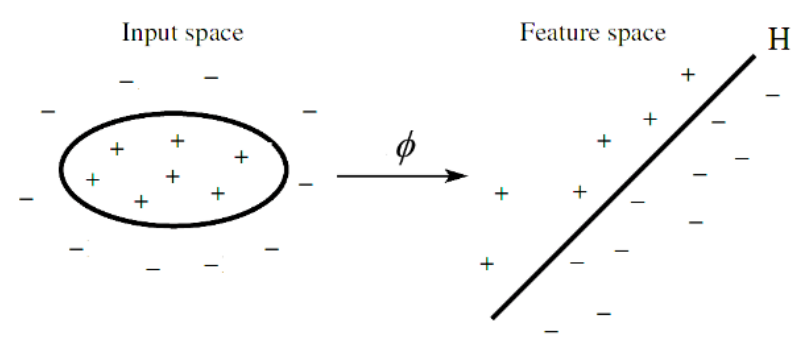

b)

Figure 1: a) maximum separation hyperplane b) classes separation in feature space

The nature of SVMs is the clarification to a classification problem pointed out by the support vectors that determine the maximum margin hyperplane. SVMs can also be used to separate classes that cannot be separated with a linear classifier (see Figure 1b, left). In such cases, the coordinates of the objects are mapped into a feature space using nonlinear functions called feature functions $\phi$. The feature space is a high-dimensional space in which the two classes can be separated with a linear classifier (see Figure $1 \mathrm{~b}$, right). The nonlinear feature function $\phi$ combines the input space (the original coordinates of the objects) into the feature space, which can even have an infinite dimension. Because the feature space is high dimensional, it is not practical to use directly feature functions $\phi$ in computing the classification hyperplane. Instead, the nonlinear mapping by the feature functions is computed with special nonlinear functions that called kernels. Kernels have the advantage of operating within the input space, where the solution of the classification problem is a weighted sum of kernel functions evaluated at the support vectors [10]. In most real-world applications data of both classes are overlapping, which makes a perfect linear separation impossible [11]. Therefore, a restricted number of misclassifications should be tolerated around the margin. The resulting optimization problem for SVMs, where the violation of the constraints is penalized, is written as

$$
\begin{array}{ll}
\text { Minimize } & J\left(w, w_{0}, \xi\right) \\
\text { such that } \xi_{i} \geq 0 & \\
w^{T} x_{i}+w_{0} \geq 1-\xi_{i} & \text { if } x_{i} \in w_{1} \\
w^{T} x_{i}+w_{0} \leq-1+\xi_{i} & \text { if } x_{i} \in w_{2}
\end{array}
$$$$
J\left(w, w_{0}, \xi\right)=\frac{1}{2}\|w\|^{2}+C \sum_{i=1}^{N} \xi_{i}
$$

where $\xi_{i} \geq 0, \mathrm{C}$ is a user-defined constant which in the cost function defines the compromise between misclassification error and a large margin. SVMs are based on the principle of structural risk minimization, which balances model complexity through regularization. The first set of constraints corresponds to $\mathrm{y}_{i}\left(w^{T} x_{i}+b\right) \geq 1$ while the second set imposes positive slack variables $\xi_{i}$, tolerating misclassifications. The value of $\xi_{i}$ indicates the distance of $x_{i}$ with respect to the decision boundary in the event of $\xi_{i}=0: x_{i}$ is correctly classified and lies outside the margin or on the margin boundary, $0<\xi_{i}<1: x_{i}$ is correctly classified, but lies inside the margin and $\xi_{i} \geq 1$ : It implies that the decision function and the target have a different sign, indicating that $x_{i}$ is misclassified [9].

\section{MATERIALS AND METHODS \\ 2.1 Subjects}

The training and testing data sets of NO concentration were obtained from fifteen lacunar stroke patients (age $65.6 \pm$ 13.61years old) and sixteen young healthy controls (age 27.33 \pm 3.85 years old). In this study, breath-holding for 30 seconds without a drop of oxygen saturation $\left(\mathrm{SaO}_{2}\right)$ were used for CVR test composing of basal, experiment and recovery phases [12]. All subject gave inform sign the consent to participate, which approved by the local ethical committee, faculty of medicine, Thammasat University, Thailand (MTUEC-PH-6-076155)

\subsection{NO measurement}

Blood sample $(3 \mathrm{ml})$ was withdrawn through a retained catheter of each experiment period. After withdrawal, whole blood was centrifuged and separated for plasma and then NO in plasma was measured by real time electrochemistry method based on the direct oxidation of NO to nitrite [13] . This electrochemistry technique is based on the nitrite level because NO is electrochemical reactive species that oxidized on the surface of carbon-fiber microelectrode (amino-700 probe, inNO-T S/N 3782-G, Tampa, Florida, USA) as shown in the following reaction.

$$
\mathrm{NO} \stackrel{-e^{-}}{\rightarrow} \frac{\mathrm{NO}^{+}}{\text {nitrosonium }} \stackrel{+\mathrm{HO}^{-}}{\rightarrow} \mathrm{HONO} \stackrel{-\mathrm{H}^{+}}{\rightarrow} \frac{\mathrm{NO}_{2}^{-}}{\text {nitrite }}
$$

The electric current $(\mathrm{pA})$ was measured and showed linear proportion to NO concentration. The standard curve was calibrated by the microelectrode probe soaked into calibration solution and set background of sensor to zero. The reaction of nitric oxide measuring system showed the proportion of nitrite and $\mathrm{NO}$ was a ratio of 1:1. Conversion factor of current (pA) to NO concentration was performed. Linear correlation between NO (nM) and current (pA) was 0.98 . The sensitivity was approximately about $5 \mathrm{nM} / \mathrm{L}$. 


\section{EXPERIMENT SETUP}

Most important of the classification algorithms or classifiers is the estimation of their best performance. The accuracy denotes the ratio of cases that is correctly classified. The experimental procedure was initiated by using SVMs for solving a problem in the classification task. Map of the original data vectors into a higher-dimensional space is applied and separable linearity of data classes will be a result. SVMs performance is depended on distinguishable kernel functions which each single kernel function reveals its different features and limitations. Consider the model, two or more single kernel functions to construct the best hybrid kernel model in order to obtain the best performance for NO evaluation are combined. Moreover, compares with the single kernel function, optimal performance of hybrid kernel function is essential because of an inappropriate parameter. Therefore, the most essential step is an exploration for the best bound of each single kernel parameter.

\subsection{Data normalization}

For data modeling, some standard procedures should be used for characterized data modeling. In order to reduce errors, the variables are normalized in the range from -1 to +1 by

$$
x_{\mathrm{i},[-1+1]}=\frac{z_{i}-\left(\frac{z_{\max }+z_{\min }}{2}\right)}{\left(\frac{z_{\max }-z_{\min }}{2}\right)}
$$

where $x_{i,[-1+1]}=$ a set of training and testing data $i$ normalized between -1 and $+1, z_{i}=$ each experimental data $i, z_{\max }=$ the maximum of all the data, and $z_{\min }=$ the minimum of all the data.

\subsection{Kernel function matching}

To consider the best kernel function that matching to a particular task, classifier performance of SVMs is introduced. Previously, many studies suggested that RBF kernel function had been proved very effective in solving problems owing to it is able to great adapt to various operations. Regarding a dynamic process of $\mathrm{NO}$ in plasma during CVR, artificial neural network (ANN) is the most machine learning that it should be applied in NO phenomena. Therefor sigmoid kernel function is proposed to be the most application. Linear SVMs was previously developed by using basic polynomial kernel function. This function is able to forecast change of $\mathrm{NO}$ in lacunar stroke [11, 14-15].

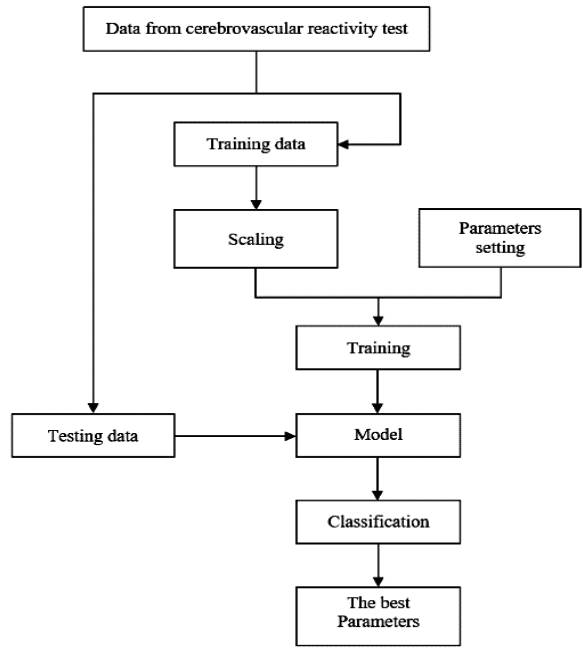

Figure 2: Experiment procedure

\subsection{An improved kernel function}

As CVR reflects multiple regulating mechanisms concerning cerebral blood flow and neurovascular function, the best fit explaining the dynamic process should be from the combination function model. In this study, a novel hybrid kernel model is proposed to be the most representative of CVR findings.

Table 1. The various types of basic single kernel functions

\begin{tabular}{|c|c|c|}
\hline $\begin{array}{c}\text { Type of kernel } \\
\text { function }\end{array}$ & $\begin{array}{c}\text { Equations of kernel } \\
\text { function }\end{array}$ & $\begin{array}{c}\text { Experiment } \\
\text { parameter }\end{array}$ \\
\hline Linear & $f_{k}(x, y)=x^{T} y+c$ & - \\
\hline RBF & $f_{k}(x, y)=\exp \left(-\frac{\|x-y\|^{2}}{2 \sigma^{2}}\right)$ & $\sigma$ \\
\hline Polynomial & $f_{k}(x, y)=\left(x^{T} y+\beta\right)^{d}$ & $\beta$ and $d$ \\
\hline Sigmoid & $f_{k}(x, y)=\tanh \left(\gamma\left(x^{T} \cdot y\right)+\alpha\right)$ & $\gamma$ and $\alpha$ \\
\hline
\end{tabular}

As a new hybrid kernel function combination, all single linear, RBF, sigmoid and polynomial kernel functions are used for training NO data afterwards. The kernel function performance is showed in Table 1 . The next procedure, single linear, RBF, sigmoid and polynomial kernels function are blended to a new mixture of each function namely hybrid kernel function. It's an initial equation that is expressed by the following:

$$
\begin{aligned}
f_{h k f}(x, y) & =\psi_{1} \exp \left(-\frac{\|x-y\|^{2}}{2 \sigma^{2}}\right)+\psi_{2}\left(x^{T} \cdot y+\beta\right)^{d} \ldots \\
& +\psi_{3} \tanh \left(\gamma\left(x^{T} y\right)+\alpha\right)
\end{aligned}
$$

Since SVMs are supervised methods, training data are performed during the experiment procedure (see Figure 2) and then the model is tested by the testing data for the best finding. An improved kernel function is constructed in three stages. First, to set up a variable by suppose $\psi_{1}=1, \psi_{2}=\psi_{3}=0$ or in other words, a single RBF kernel function and parameter are chosen, then $\sigma$ is selected for further running. Then, by simulation, experiments could be the performance of SVMs with the trend of changes, thus, the best parameter $\sigma$ of the RBF kernel can be chosen. The second and third stages, the best parameter $\beta$ and $d$ of the polynomial and the best parameter $\gamma$ and $\alpha$ of the sigmoid kernel function by suppose that $\psi_{1}=\psi_{3}=0, \psi_{2}=1$ and $\psi_{1}=\psi_{2}=0, \psi_{3}=1$ are selected. Then, the best parameters have been identified $(\beta, b, \sigma, \rho$ and $\alpha$ ) to the hybrid kernel function. The proportion of parameters $\left(\psi_{l}\right.$, 
$\psi_{2}$ and $\psi_{3}$ ) are applied to initial equation (in Eq.10) of each running cycle in order to obtain the best performance. From the results, the best parameters are used to construct the hybrid kernel function for training the samples. The hybrid kernel function is

$$
f_{h k f}(x, y)=\psi\left(x^{T} \cdot y+1\right)^{3}+\lambda\left(\frac{e^{2\left(x^{T} \cdot y+3\right)}+1}{e^{2\left(x^{T} \cdot y+3\right)}+1}\right)
$$

where $\psi$ and $\lambda$ are user-defined parameters. The values of the parameter $(\psi$ and $\lambda)$ are varied to train and test the data sets, the best parameters can be determined from their finding result.

\section{RESULTS}

NO levels in healthy control and lacunar stroke are shown (see Table 2). During basal, experiment and recovery phases, NO in each phase was tested for comparison of classifier performance of each kernel function (see Tables 3-5)

\section{Table 2. The data of NO concentration in participants}

\begin{tabular}{|c|c|c|c|}
\hline $\begin{array}{c}\text { Experiment } \\
\text { Phases }\end{array}$ & & \multicolumn{2}{|c|}{ NO concentration (nM/L) } \\
\cline { 3 - 4 } Basal & Max & 82.20 & stroke \\
\hline \multirow{3}{*}{ Experiment } & Min & 23.30 & 67.40 \\
\cline { 2 - 4 } & Mean \pm SD & $\underline{58.06 \pm 19.63}$ & $\underline{39.00 \pm 14.11}$ \\
\cline { 2 - 4 } & Max & 105.30 & 63.60 \\
\cline { 2 - 4 } & Min & 78.00 & 18.20 \\
\cline { 2 - 4 } & Mean \pm SD & $\underline{73.11 \pm 18.56}$ & $\underline{40.21 \pm 14.94}$ \\
\hline \multirow{3}{*}{ Recovery } & Max & 98.00 & 68.8 \\
\cline { 2 - 4 } & Min & 28.40 & 24.6 \\
\cline { 2 - 4 } & Mean \pm SD & $63.63 \pm 20.85$ & $38.68 \pm 15.61$ \\
\hline
\end{tabular}

\subsection{Classifier performance of nitric oxide} (NO) by each single kernel function

\subsubsection{Polynomial kernel function for $\mathrm{NO}$} assessment

Table 3. A classifier performance of NO by polynomial kernel function in each phase

\begin{tabular}{|c|c|c|c|}
\hline \multirow[b]{2}{*}{ Phase } & \multicolumn{3}{|c|}{ Polynomial kernel functions } \\
\hline & Parameter $^{\dagger}$ & $\begin{array}{c}\text { Classified } \\
\text { accuracy }(\%)\end{array}$ & $\begin{array}{l}\text { No. support } \\
\text { vectors }\end{array}$ \\
\hline \multirow{5}{*}{ Basal } & $\beta=1, d=2$ & $88.00 *$ & 7 \\
\hline & $\beta=1, d=5$ & 76.00 & 18 \\
\hline & $\beta=5, d=2$ & 78.00 & 36 \\
\hline & $\beta=1, d=3$ & 86.00 & 9 \\
\hline & $\beta=1, d=4$ & 74.00 & 28 \\
\hline \multirow{5}{*}{ Experiment } & $\beta=1, d=5$ & 80.00 & 8 \\
\hline & $\beta=5, d=2$ & 78.00 & 27 \\
\hline & $\beta=3, d=3$ & $90.00 *$ & 9 \\
\hline & $\beta=3, d=4$ & 78.00 & 7 \\
\hline & $\beta=4, d=4$ & 86.00 & 33 \\
\hline \multirow{5}{*}{ Recovery } & $\beta=1, d=5$ & $84.00 *$ & 8 \\
\hline & $\beta=5, d=2$ & 78.00 & 35 \\
\hline & $\beta=2, d=3$ & 82.00 & 16 \\
\hline & $\beta=5, d=3$ & 72.00 & 14 \\
\hline & $\beta=3, d=4$ & 76.00 & 33 \\
\hline
\end{tabular}

*Significant notice of high accuracy of kernel function. ${ }^{\top}$ Parameter can be considered by Table 1

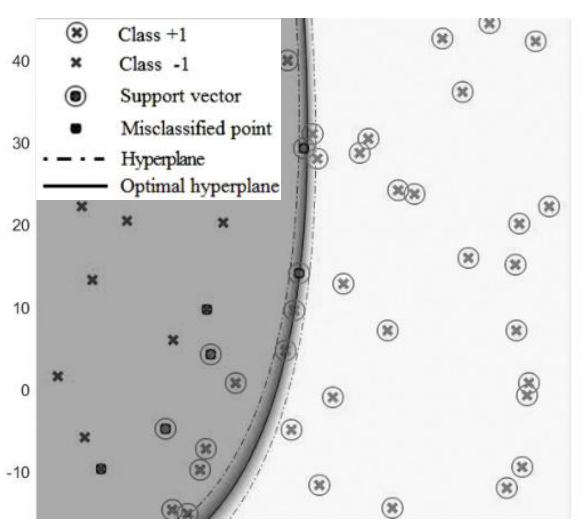

a)

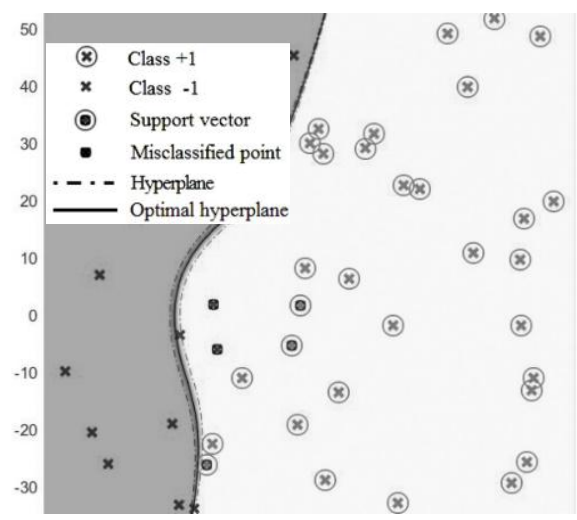

b)

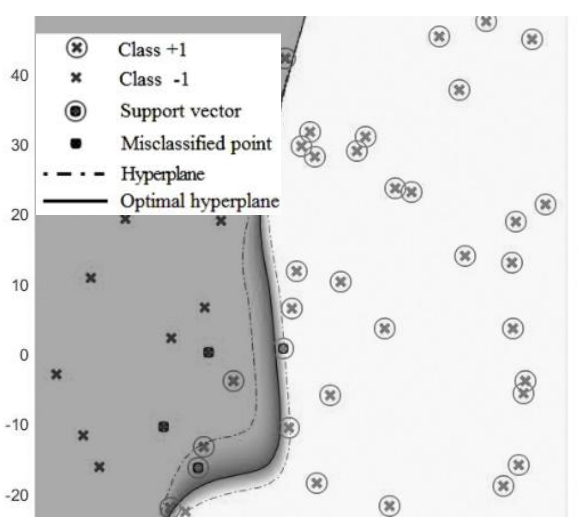

c)

Figure 3: Plot of NO classification by polynomial kernel function in a) basal phase b) experiment phase and c) recovery phase

From experiment data, a highest classified accuracy is 90.00 from the polynomial in experiment phase and is followed by 88.00 and 84.00 in recovery and basal phases, respectively. According to the best parameters of polynomial kernel functions in each phase, it is able to construct the new high performance kernel function for training the samples data as well. Considering the best parameters in Table 3 and most experimental results, classifier performance of $\mathrm{NO}$ by polynomial kernel function is high when the parameter $\beta$ is odd numbers. 
4.1.2 RBF kernel function for NO assessment Table 4. A classifier performance of NO by RBF kernel function in each phase

\begin{tabular}{|c|c|c|c|}
\hline \multirow{3}{*}{ Phase } & \multicolumn{3}{|c|}{ RBF kernel functions } \\
\cline { 2 - 4 } & Parameter $^{\dagger}$ & $\begin{array}{c}\text { Classified } \\
\text { accuracy (\%) }\end{array}$ & $\begin{array}{c}\text { No. support } \\
\text { vectors }\end{array}$ \\
\hline \multirow{4}{*}{ Basal } & $\sigma=2$ & 66.00 & 50 \\
\cline { 2 - 4 } & $\sigma=3$ & 76.00 & 50 \\
\cline { 2 - 4 } & $\sigma=4$ & 78.00 & 44 \\
\cline { 2 - 4 } & $\sigma=5$ & 84.00 & 39 \\
\hline \multirow{4}{*}{ Experiment } & $\sigma=6$ & $88.00^{*}$ & 32 \\
\cline { 2 - 4 } & $\sigma=1$ & 92.00 & 50 \\
\cline { 2 - 4 } & $\sigma=4$ & $94.00^{*}$ & 42 \\
\cline { 2 - 4 } & $\sigma=7$ & 90.00 & 34 \\
\hline \multirow{4}{*}{ Recovery } & $\sigma=15$ & 90.00 & 26 \\
\cline { 2 - 4 } & $\sigma=1$ & 88.00 & 23 \\
\cline { 2 - 4 } & $\sigma=2$ & 76.00 & 50 \\
\cline { 2 - 4 } & $\sigma=3$ & 80.00 & 50 \\
\cline { 2 - 4 } & $\sigma=4$ & 82.00 & 50 \\
\hline & $\sigma=8$ & $84.00 *$ & 31 \\
\hline
\end{tabular}

*Significant notice of high accuracy of kernel function. ${ }^{\dagger}$ Parameter can be considered by Table 1

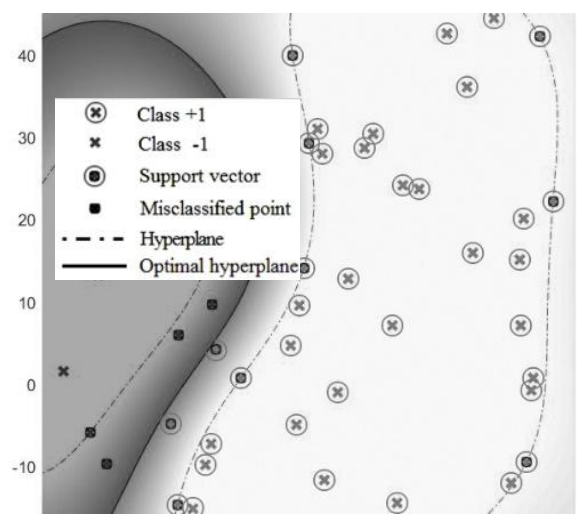

a)

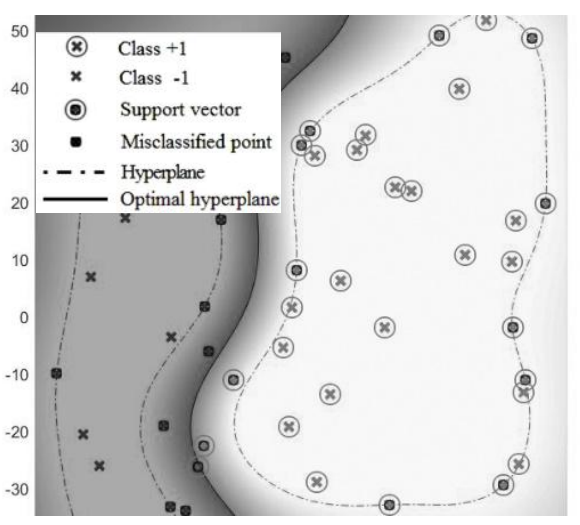

b)

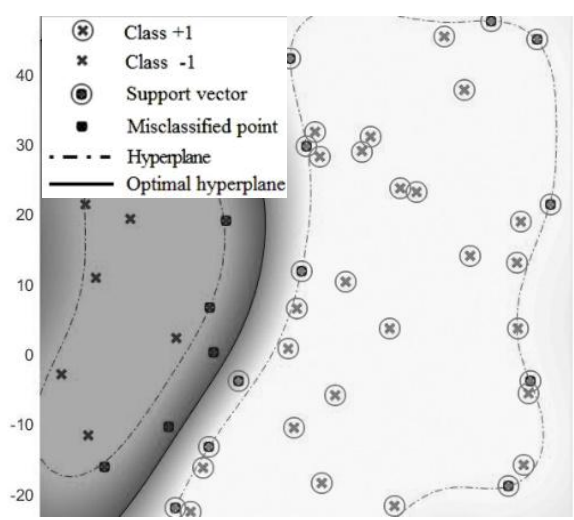

c)

Figure 4: Classification of NO assessed by RBF kernel function in a) basal phase b) experiment phase and c) recovery phase

All findings studied by RBF kernel function, the classification result of RBF in experiment phase is the best, followed by the RBF kernel function in basal and recovery phase, respectively. Then the parameter $\sigma$ are chosen to test the sample;the optimal parameters present in Table 4 and Figure 4 and show the separate results from the kernel function. The classifier performance of NO by RBF kernel is high when the parameter $\sigma$ is an even number.

4.1.3 Sigmoid kernel function for NO assessment Table 5. A classifier performance of NO by Sigmoid kernel function in each phase

\begin{tabular}{|c|c|c|c|}
\hline \multirow[b]{2}{*}{ Phase } & \multicolumn{3}{|c|}{ Sigmoid kernel functions } \\
\hline & Parameter $^{\dagger}$ & $\begin{array}{c}\text { Classified } \\
\text { accuracy }(\%)\end{array}$ & $\begin{array}{l}\text { No. support } \\
\text { vectors }\end{array}$ \\
\hline \multirow{5}{*}{ Basal } & $\gamma=1, \alpha=1$ & $74.00 *$ & 26 \\
\hline & $\gamma=1, \alpha=2$ & 66.00 & 29 \\
\hline & $\gamma=1, \alpha=4$ & 68.00 & 24 \\
\hline & $\gamma=2, \alpha=2$ & 72.00 & 32 \\
\hline & $\gamma=2, \alpha=3$ & 68.00 & 30 \\
\hline \multirow{5}{*}{ Experiment } & $\gamma=1, \alpha=2$ & 54.00 & 8 \\
\hline & $\gamma=1, \alpha=3$ & $58.00 *$ & 12 \\
\hline & $\gamma=1, \alpha=5$ & 56.00 & 45 \\
\hline & $\gamma=3, \alpha=3$ & 54.00 & 27 \\
\hline & $\gamma=3, \alpha=5$ & 52.00 & 16 \\
\hline \multirow{5}{*}{ Recovery } & $\gamma=1, \alpha=2$ & $78.00 *$ & 26 \\
\hline & $\gamma=1, \alpha=4$ & 58.00 & 26 \\
\hline & $\gamma=2, \alpha=1$ & 46.00 & 27 \\
\hline & $\gamma=2, \alpha=3$ & 52.00 & 26 \\
\hline & $\gamma=2, \alpha=4$ & 48.00 & 27 \\
\hline
\end{tabular}

*Significant notice of high accuracy of kernel function.

Parameter can be considered by Table 1 


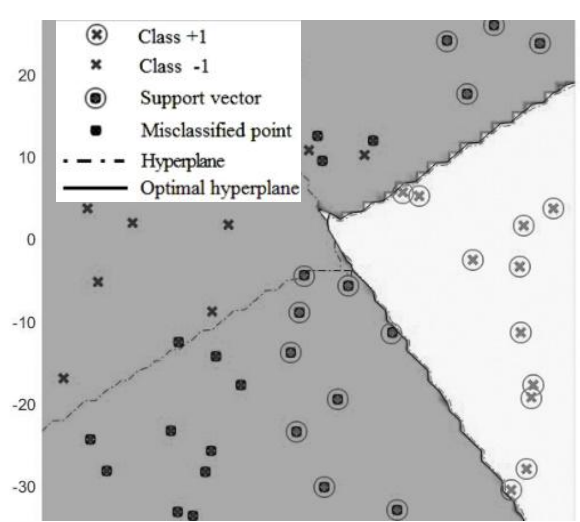

a)

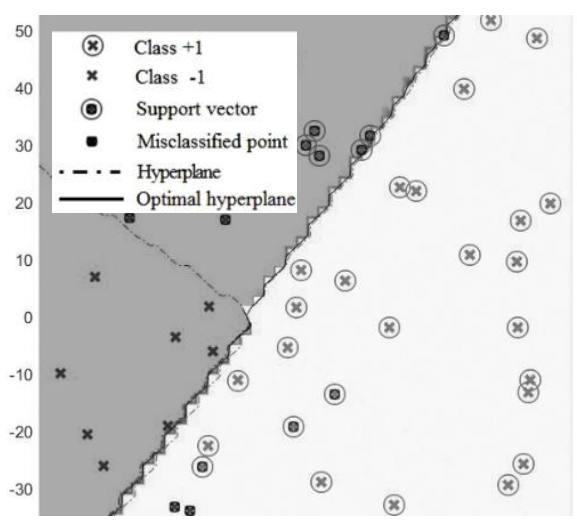

b)

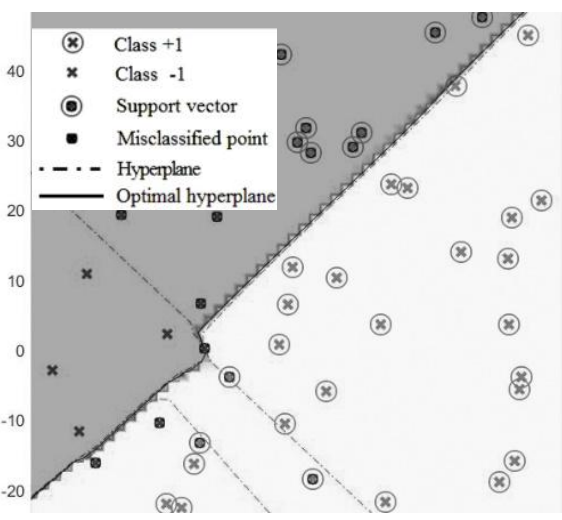

c)

Figure 5: Classification of NO assessed by sigmoid kernel function in a) basal phase b) experiment phase and c) recovery phase

From experiment results, a highest classified accuracy is 78.00 in recovery phase and is followed by 74.00 and 58.00 in basal and experiment phase, respectively. Considering the best parameters in Table 5 and Figure 5, the results have shown that classifier performance of NO by sigmoid kernel function is high when the sum of a parameter $\gamma$ and $\alpha$ is odd numbers.

\subsection{An enhancement of classifier performance of NO assessment in each phase by hybrid kernel function}

4.2.1 Hybrid kernel function for NO assessment Table 6. A classifier performance of NO by hybrid kernel function in each phase

\begin{tabular}{|c|c|c|c|}
\hline \multirow{4}{*}{ Phase } & \multicolumn{3}{|c|}{ Hybrid kernel functions } \\
\cline { 2 - 4 } & Parameter $^{\dagger}$ & $\begin{array}{c}\text { Classified } \\
\text { accuracy (\%) }\end{array}$ & $\begin{array}{c}\text { No. support } \\
\text { vectors }\end{array}$ \\
\hline \multirow{5}{*}{ Basal } & $\psi=1, \lambda=3$ & 82.00 & 19 \\
\cline { 2 - 4 } & $\psi=1, \lambda=4$ & 86.00 & 18 \\
\cline { 2 - 4 } & $\psi=1, \lambda=5$ & 88.00 & 11 \\
\cline { 2 - 4 } & $\psi=0.1, \lambda=0.4$ & 90.00 & 6 \\
\cline { 2 - 4 } & $\psi=0.5, \lambda=0.5$ & $94.00^{*}$ & 6 \\
\hline \multirow{5}{*}{ Experiment } & $\psi=1, \lambda=1$ & 92.00 & 16 \\
\cline { 2 - 4 } & $\psi=0.1, \lambda=0.1$ & 92.00 & 14 \\
\cline { 2 - 4 } & $\psi=0.1, \lambda=0.3$ & 92.00 & 15 \\
\cline { 2 - 4 } & $\psi=0.3, \lambda=0.5$ & 90.00 & 12 \\
\cline { 2 - 4 } & $\psi=0.4, \lambda=0.5$ & $96.00^{*}$ & 17 \\
\hline \multirow{5}{*}{ Recovery } & $\psi=1, \lambda=4$ & 78.00 & 15 \\
\cline { 2 - 4 } & $\psi=0.1, \lambda=0.2$ & 76.00 & 14 \\
\cline { 2 - 4 } & $\psi=0.1, \lambda=0.5$ & 80.00 & 11 \\
\cline { 2 - 4 } & $\psi=0.3, \lambda=0.5$ & 92.00 & 7 \\
\cline { 2 - 4 } & $\psi=0.5, \lambda=0.5$ & $92.00^{*}$ & 4 \\
\hline
\end{tabular}

* Significant notice of high accuracy of kernel function. Parameter can be considered by Table 1 and Eq.11

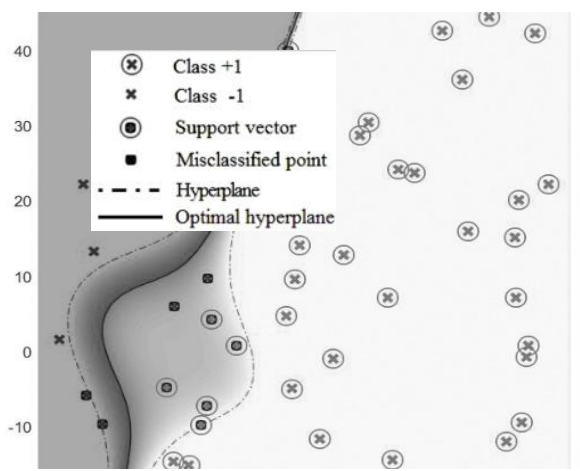

a)

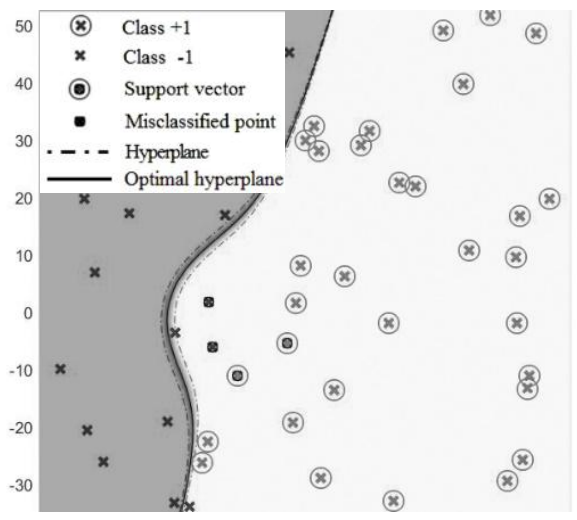

b) 


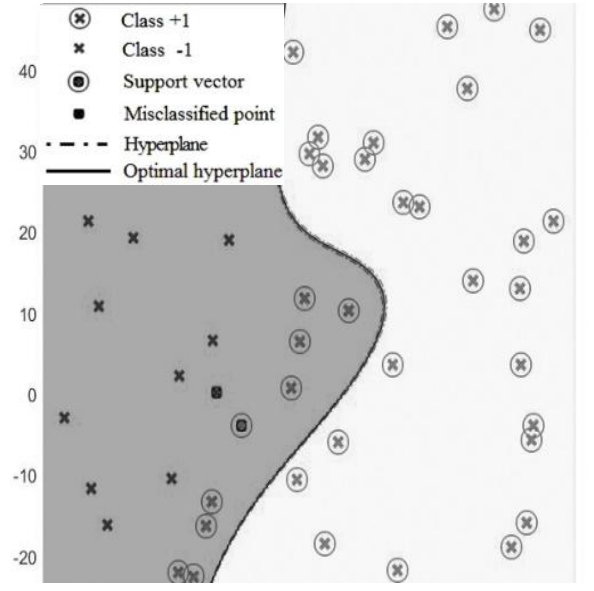

c)

Figure 6: Classification of NO assessed by hybrid kernel function in a) basal phase b) experiment phase and c) recovery phase

Figure 6 shows the separate results of the hybrid kernel function. The value of a parameter ( $\psi$ and $\lambda$ ) is varied to test the data sets in each phrase, the best parameters determined are present in Table 6 . The best values of both parameters ( $\psi$ and $\lambda$ ) resulting in the highest performance for classification of nitric oxide for hybrid kernel function are 0.5. Consider the values of parameters compared with highest performance, low value of parameters is detected.

An improvement of nitric oxide classifier performance: According to the previous experiments, the best parameters of each kernel functions are used to construct the novel hybrid kernel function for improvement of classifier performance as showed in Table 6 and summarized in Table 7.

Table 7. Summary of the best results of each model

\begin{tabular}{|c|c|c|}
\hline Phase & $\begin{array}{c}\text { The best } \\
\text { parameter }\end{array}$ & $\begin{array}{c}\text { The best classification } \\
\text { modeling }\end{array}$ \\
\hline \multirow{4}{*}{ Basal } & $\begin{array}{l}\text { Polynomial: } \\
\beta=1, d=2\end{array}$ & $\left(x^{T} \cdot y+1\right)^{2}$ \\
\hline & $\begin{array}{l}\mathrm{RBF} \\
\sigma=6\end{array}$ & $\exp \left(-\frac{\|x-y\|^{2}}{2(6)^{2}}\right)$ \\
\hline & $\begin{array}{c}\text { Sigmoid: } \\
\gamma=1, \beta=1\end{array}$ & $\tanh \left(\left(x^{T} \cdot y\right)+1\right)$ \\
\hline & $\begin{array}{l}\text { Hybrid } \\
\psi=0.5, \\
\lambda=0.5\end{array}$ & $0.5\left(x^{T} \cdot y+1\right)^{3}+0.5\left(\frac{e^{2\left(x^{T} \cdot y+3\right)}+1}{e^{2\left(x^{T} \cdot y+3\right)}+1}\right)$ \\
\hline \multirow{4}{*}{ Experiment } & $\begin{array}{l}\text { Polynomial: } \\
\beta=3, d=3\end{array}$ & $\left(x^{T} \cdot y+1\right)^{3}$ \\
\hline & $\begin{array}{l}\text { RBF: } \\
\sigma=4\end{array}$ & $\exp \left(-\frac{\|x-y\|^{2}}{2(4)^{2}}\right)$ \\
\hline & $\begin{array}{c}\text { Sigmoid: } \\
\gamma=1, \beta=3\end{array}$ & $\tanh \left(\left(x^{T} \cdot y\right)+3\right)$ \\
\hline & $\begin{array}{l}\text { Hybrid: } \\
\psi=0.4, \\
\lambda=0.5\end{array}$ & $0.4\left(x^{T} \cdot y+1\right)^{3}+0.5\left(\frac{e^{2\left(x^{T} \cdot y+3\right)}+1}{e^{2\left(x^{T} \cdot y+3\right)}+1}\right)$ \\
\hline Recovery & Polynomial: & $\left(x^{T} \cdot y+1\right)^{5}$ \\
\hline
\end{tabular}

\begin{tabular}{|c|c|l|}
\hline \multirow{1}{*}{} & $\beta=1, d=5$ & \\
\cline { 2 - 3 } & $\mathrm{RBF}:$ & $\exp \left(-\frac{\|x-y\|^{2}}{2(8)^{2}}\right)$ \\
$\sigma=8$ & $\tanh \left(\left(x^{T} \cdot y\right)+2\right)$ \\
\cline { 2 - 3 } & Sigmoid: & \\
& $\gamma=1, \beta=2$ & \\
\cline { 2 - 3 } & Hybrid: & \multirow{2}{*}{$0.5\left(x^{T} \cdot y+1\right)^{3}+0.5\left(\frac{e^{2\left(x^{T} \cdot y+3\right)}+1}{e^{2\left(x^{T} \cdot y+3\right)}+1}\right)$} \\
& $\psi=0.5$, \\
$\lambda=0.5$ & \\
\hline
\end{tabular}

\section{DISCUSSION}

Potential hybrid model of kernel function is proposed to be the most the highest classifier accuracies of NO in healthy control and lacunar stroke during basal, experiment and recovery phases are $94 \%, 96 \%$ and $92 \%$, respectively. According to pathophysiology of small deep arteriole occlusion caused from endothelial dysfunction and vascular inflammation, NO is slightly reduced compared to healthy control in basal phase. During CVR, small change of NO is evident in experiment and recovery phases in patient, but they differ from those in control. Greatest classifier model of hybrid kernel function reflects the mechanism of LS, even though, in clinical view, LS is considered as minor stroke since it shows minor neurological deficiency. In other words, CVR is the best method testing cerebral reserve function and autoregulation in LS which it is confirmed by high classifier accuracy among basal, experiment and recovery phases.

\section{CONCLUSIONS}

The performance of SVMs depends on the different kind of kernel functions and adjustment of its parameters because each single kernel function represents own limitation of each different feature. Concerning NO availability, the best model explaining a dynamic process should be a nonlinear model. Look at the process, two or more single kernel functions are combined and develop hybrid kernel function which it fits with NO data during CVR. On the other hand, the performance of the hybrid kernel function would be worse than the single ones because of an excessive parameter combination. Therefore, the critical step is searching for the optimal form of each parameter of single kernel function. It is suitable for distinguished change in lower NO levels in high risk of stroke and lacunar infarction. These results will be applied and monitored higher risk of acute stroke such as atrial fibrillation.

\section{ACKNOWLEDGMENTS}

This work was supported by National Research University Project of Thailand, Office of Higher Education Commission and faculty of medicine, Thammasat University.

\section{REFERENCES}

[1] C. M. Fisher, "Lacunar strokes and infarcts: A review," Neurology, vol. 32, pp. 871-876, 1982.

[2] C. Jackson and C. Sudlow, "Comparing risks of death and recurrent vascular events between lacunar and nonlacunar infarction", Brain, vol. 128, pp. 2507-2517, 2005.

[3] N. Schwertfeger, P. Neu, P. Schlattmann, H. Lemke, I. Heuser, and M. Bajbouj, "Cerebrovascular reactivity over time course in healthy subjects", Journal of the Neurological Sciences, vol. 249, pp. 135-139, 2006. 
[4] K. Intharakham and K. Suwanprasert, "Complexity of autonomic control during cerebrovascular reactivity", in BMEiCON 2013 - 6th Biomedical Engineering International Conference, 2013.

[5] S. Lavi, R. Egbarya, R. Lavi, and G. Jacob, "Role of nitric oxide in the regulation of cerebral blood flow in humans: Chemoregulation versus mechanoregulation", Circulation, vol. 107, pp. 1901-1905, 2003.

[6] E. D. Gommer, J. Staals, R. J. Van Oostenbrugge, J. Lodder, W. H. Mess, and J. P. H. Reulen, "Dynamic cerebral autoregulation and cerebrovascular reactivity: A comparative study in lacunar infarct patients", Physiological Measurement, vol. 29, pp. 1293-1303, 2008 .

[7] M. N. Wernick, Y. Yang, J. G. Brankov, G. Yourganov, and S. C. Strother, "Machine Learning in Medical Imaging", IEEE signal processing magazine, vol. 27, pp. 25-38, 2010

[8] R. A. Berk, "Support Vector Machines", in Statistical Learning from a Regression Perspective, ed New York, NY: Springer New York, 2008, pp. 1-28.

[9] Y. Pimtongngam, K. Intharakham, and K. Suwanprasert, "Classification of nitric oxide assessed by hybrid kernel function in lacunar stroke", in BMEiCON 2013 - 6th Biomedical Engineering International Conference, 2013.
[10] H. Li, Y. Liang, and Q. Xu, "Support vector machines and its applications in chemistry", Chemometrics and Intelligent Laboratory Systems, vol. 95, pp. 188-198, 2009.

[11] J. Luts, F. Ojeda, R. Van de Plas, B. De Moor, S. Van Huffel, and J. A. K. Suykens, "A tutorial on support vector machine-based methods for classification problems in chemometrics", Analytica Chimica Acta, vol. 665 , pp. $129-145,4 / 30 / 2010$.

[12] K. Intharakham and K. Suwanprasert, " Neurovascular Coupling assessed by Heart Rate Variability (HRV), Circulating Nitric Oxide and Hydrogen Peroxide during Cerebrovascular Reactivity", in IBRO-APRC 2013 - 17th Thai Neuroscience Society International Conference, 2013.

[13] Y. C. Boo, S. L. Tressel, and H. Jo, "An improved method to measure nitrate/nitrite with an NO-selective electrochemical sensor", Nitric Oxide - Biology and Chemistry, vol. 16, pp. 306-312, 2007.

[14] O. Chapelle, V. Vapnik, O. Bousquet, and S. Mukherjee, "Choosing Multiple Parameters for Support Vector Machines," Machine Learning, vol. 46, pp. 131-159, 2002.

[15] A. Rakotomamonjy, "Variable selection using SVM based criteria", J. Mach. Learn. Res., vol. 3, pp. 13571370, 2003 